\title{
SISTEMA DE AUTORREGULACIÓN PUBLICITARIA: DEL COMPROMISO ÉTICO AL CONTROL EFECTIVO DE LA PUBLICIDAD EN ESPAÑA
}

\author{
The advertising self-regulation system: From ethical \\ commitment to effective control over \\ advertising in Spain
}

\section{Sandra Vilajoana-Alejandre y Josep Rom-Rodríguez}
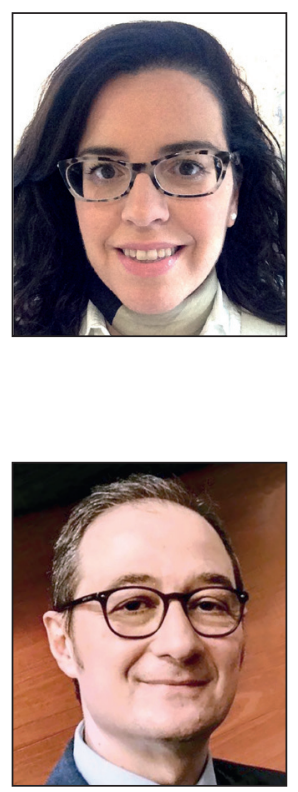

Sandra Vilajoana-Alejandre es profesora de los Estudios de Ciencias de la Información y de la Comunicación en la Universitat Oberta de Catalunya e imparte la asignatura Derecho de la comunicación en la Facultad de Comunicación y Relaciones Internacionales Blanquerna de la Universitat Ramon Llull (URL). Es doctora en comunicación y humanidades y licenciada en publicidad y relaciones públicas por la URL, y licenciada en derecho por la Universitat de Barcelona. Su principal línea de investigación aborda los límites jurídicos y éticos de la publicidad.

http://orcid.org/0000-0001-6231-1021

Universitat Oberta de Catalunya Estudios de Ciencias de la Información y de la Comunicación Av. Tibidabo, 39-43. 08035 Barcelona, España svilajoana@uoc.edu

Josep Rom-Rodríguez es profesor titular de Publicidad en la Facultad de Comunicación y Relaciones Internacionales Blanquerna, de la Universitat Ramon Llull (URL) y doctor en publicidad y relaciones por la URL. Es investigador principal del Grupo de Investigación en Estrategia y Creatividad en Publicidad y Relaciones Públicas y es autor de numerosas publicaciones sobre estrategia, diseño y lenguajes publicitarios. Ha dirigido una docena de tesis doctorales sobre publicidad, relaciones públicas, diseño gráfico y branding.

http://orcid.org/0000-0002-4910-2169

Universitat Ramon Llull Facultat de Comunicació i Relacions Internacionals Blanquerna Plaza Joan Coromines, 08001 Barcelona, España joseprr@blanquerna.url.edu

\section{Resumen}

La publicidad es una actividad tradicionalmente cuestionada, tanto desde el punto de vista ético como legal. Este artículo analiza el compromiso ético de la industria publicitaria mediante el estudio de las características y actividad de Autocontrol, el sistema de autorregulación publicitaria español, y la realización de entrevistas en profundidad a directivos de las principales agencias de publicidad que operan en España. Los resultados de la investigación constatan el notable compromiso ético de los profesionales de la publicidad y la relevante actividad de Autocontrol: la mayor parte de la publicidad difundida en España es objeto de una revisión ética previa a través del servicio de Copy Advise ${ }^{\circledR}$.

\section{Palabras clave}

Ética; Publicidad; Autorregulación; Agencias; Profesión; Comunicación; España; Autocontrol.

\section{Abstract}

Advertising is a traditionally questioned activity, not only from the ethical point of view, but also legal. This article analyzes the ethical commitment of the advertising industry through the study of the characteristics and activity of Autocontrol, the Spanish advertising self-regulation system, and by means of in-depth interviews to directors of the main advertising agencies operating in Spain. The results of the research show significant ethical commitment from advertising professionals and the relevant activity of Autocontrol; most of the advertising disseminated in Spain has been subject to a previous ethical review through the service of Copy Advise ${ }^{\circledR}$.

\section{Keywords}

Ethics; Advertising; Self-regulation; Agencies; Profession; Communication; Spain; Autocontrol. 
Vilajoana-Alejandre, Sandra; Rom-Rodríguez, Josep (2017). "Sistema de autorregulación publicitaria: del compromiso ético al control efectivo de la publicidad en España”. El profesional de la información, v. 26, n. 2, pp. $192-200$.

https://doi.org/10.3145/epi.2017.mar.05

\section{Introducción}

La publicidad tiene un marcado carácter social. Su influencia en los hábitos de consumo, gustos, modos de vida y actitudes de la cultura post industrial (Lipovetsky, 1994) así lo corroboran. Desde sus orígenes ha sido objeto de debate y controversia puesto que algunos sectores sociales la cuestionan desde un punto de vista sobre todo ético, pero también legal. El desarrollo teórico de la publicidad va unido a una singular circunstancia: su origen vinculado en ocasiones a ciertas mercancías fraudulentas, al charlatanismo, a la exageración y al engaño (Eguizábal, 2010). Y ello a pesar de que es una de las actividades económicas más reguladas: cerca de trescientas normas configuran el régimen jurídico de la publicidad en España (Lema-Devesa; Gómez-Montero, 2010).

El profesional de la publicidad tiene por misión persuadir al consumidor para que adquiera un producto o servicio y, precisamente, en los límites en que debe utilizar la persuasión reside la ética de su profesión (Sánchez-Guzmán, 1993). La ética y la deontología exigen al buen profesional que no busque solamente cumplir sus cometidos con destreza técnica, limitándose al estricto cumplimiento de la ley, sino que vaya más allá y trate de hacer el bien a la sociedad con su trabajo profesional. En publicidad,

"profesionalidad y ética son inseparables. El conocimiento del oficio y su recto ejercicio -que nunca olvida el bien común- constituyen el primer escalón de la ética publicitaria" (Megías-Quirós; Cabrera-Caro, 2013, p. 17).

Sin embargo, vivimos en un mundo saturado de marcas y productos en el que la competencia es acusada. En este contexto el propio instinto de supervivencia o el deseo de ganancia fácil pueden provocar conductas deshonestas, de modo que la falta de ética en el mercado se puede convertir en una falta de ética en la publicidad (Aznar, 2000). Para evitar estas conductas, los agentes implicados pueden evidenciar su compromiso ético por vía de la autorregulación.

La relación entre publicidad y ética ha sido abordada por estudios de carácter general (Boddewyn, 1989; Aznar, 2000; Martín-Llaguno; Hernández-Ruiz, 2009; 2010; Megías-Quirós; Cabrera-Caro, 2013) y por publicaciones que se han centrado en el análisis del sistema de autorregulación publicitaria español (Taylor, 2002; Patiño-Alves, 2007; Fernando-Magarzo, 2008; Muela-Molina; PerellóOliver, 2014), o en su reconocimiento, fomento y regulación (Gómez-Castallo; Fernando-Magarzo, 2010; Massaguer-Fuentes, 2011).

A pesar del interés que suscita la ética en la investigación sobre publicidad (Hyman; Tansey; Clark, 1994), todavía se observan algunas carencias en áreas importantes
(Drumwright; Murphy, 2009), como es el caso del estudio del compromiso y gestión práctica del condicionante ético por parte de las agencias. Trabajos como los de Kugman y Ferrell (1981), y Drumwright y Murphy (2004) han dado voz a los profesionales de las agencias norteamericanas para conocer sus percepciones y comportamiento ético, pero sin llegarlos a relacionar con los sistemas de autorregulación.

\section{Objetivos y metodología}

El presente artículo responde a dos objetivos:

- examinar y evaluar el compromiso ético de la industria publicitaria mediante el estudio de las características y actividad del sistema de autorregulación publicitaria español: ¿en qué consiste y sobre la base de qué evidencias se ha valorado su efectividad?;

- ante la inexistencia de trabajos previos, el segundo objetivo es conocer y constatar cómo gestionan las principales agencias de publicidad que operan en España la adecuación ética y legal de su actividad: ¿es el sistema de autorregulación publicitaria su principal referencia y hasta qué punto se traduce en un verdadero compromiso ético de la profesión?

Se emplea una metodología cualitativa que combina el trabajo de gabinete -revisión de la literatura-con el trabajo de campo -entrevistas en profundidad a directivos de agencias de publicidad-.

Más allá de la legalidad, los profesionales de la publicidad evidencian su compromiso ético por vía de la autorregulación

Las principales agencias publicitarias que operan en España configuraron el universo inicial del estudio, a fin de conformar una muestra de los modelos de agencia que gestionan un mayor volumen de la inversión publicitaria controlada por Infoadex. A continuación se delimitó una muestra ilustrativa -no probabilística- constituida por las 20 primeras agencias por volumen de inversión según el Estudio de InfoAdex de agencias de publicidad 2014, cuyos datos recogen la inversión gestionada en 2013 (Ipmark, 2014).

Las cuestiones éticas y legales suelen estar sujetas a compromisos de confidencialidad e incidir en la propia reputación de la agencia. Debido a ello, el tamaño de la muestra se tuvo que reformular en el proceso de investigación y se redujo finalmente a ocho agencias, al declinar el resto participar en el estudio. La muestra final coincide con los entre seis y ocho informantes que se requieren para que esta sea homogénea (Daymon; Halloway, 2011). 
Tabla 1. Configuración muestra final entrevistas

\begin{tabular}{|c|l|l|l|c|}
\hline $\begin{array}{c}\text { Ranking } \\
\mathbf{2 0 1 3}\end{array}$ & \multicolumn{1}{|c|}{ Agencia } & \multicolumn{1}{|c|}{ Entrevistado/a } & \multicolumn{1}{c|}{ Cargo } & $\begin{array}{c}\text { Ranking } \\
\mathbf{2 0 1 5}\end{array}$ \\
\hline 1 & McCann & Gonzalo Sánchez-Táiz & Director general ejecutivo & 1 \\
\hline 5 & TBWA & $\begin{array}{l}\text { Nicole Moller } \\
\text { Nacho Hortal }\end{array}$ & $\begin{array}{l}\text { Directora general } \\
\text { Director de cuentas }\end{array}$ & 6 \\
\hline 8 & Sra. Rushmore & Clemente Manzano & Director general & 4 \\
\hline 9 & JWT & Manuela Cisneros & Directora financiera & 11 \\
\hline 10 & Leo Burnett & Clara Marchan & Directora general & 9 \\
\hline 14 & $*$ S,C,P,F & Helena Grau & Directora servicios cliente & 12 \\
\hline 18 & Barbara & Belén Romero & Socia & 18 \\
\hline 19 & Tiempo BBDO & Julio Paredes & Director general & 21 \\
\hline
\end{tabular}

bación de normas jurídicas, de obligado cumplimiento, con el objetivo de garantizar que la publicidad alcance sus fines sin perjuicio de otros derechos, como la protección de los consumidores o la libre competencia. Pero más allá de la legalidad, los publicistas pueden evidenciar su compromiso ético por la vía de la autorregulación.

La autorregulación publicitaria es la regulación llevada a cabo por los propios sujetos que participan activamente en la realización, creación, preparación, ejecución y difusión de

En la tabla 1 se indica la posición de cada agencia en el Estudio Infoadex de agencias de publicidad 2014 -y también, su posición en la última versión del mismo, publicada a finales de 2016 (Ipmark, 2016) - y la identificación de las agencias y los profesionales entrevistados, mayoritariamente directores generales y los responsables de otras direcciones a las que éstos nos derivaron. Las entrevistas se llevaron a cabo presencialmente, en las oficinas de las agencias en Madrid y en Barcelona, entre los meses de enero y octubre de 2015, y todos los participantes autorizaron su identificación en el estudio.

Las ocho entrevistas en profundidad, semiestructuradas, breves, de una duración aproximada de 45 minutos, fueron grabadas previa autorización de los entrevistados. La técnica de la entrevista en profundidad es especialmente adecuada cuando los entrevistados son difícilmente accesibles -entre otras cuestiones, por razón de cargo- y los temas a tratar son difíciles o comprometidos, como era el caso (Vilches, 2011). Sobre la base de los objetivos de la investigación se configuró el guion de la entrevista. Al ser la autorregulación una disciplina directamente relacionada con la ética y también con la regulación (Martín-Llaguno; Hernández-Ruiz, 2009), el guion contempló la posibilidad de que su gestión profesional estuviera relacionada. La observancia de los límites legales entendemos que es un presupuesto necesario aunque no suficiente para constatar el compromiso ético de la profesión. Tras un proceso de transcripción minuciosa de las entrevistas, se aplicó la técnica de análisis de contenido, con el objetivo de identificar categorías y patrones y, finalmente, interpretar y presentar los resultados.

\section{Resultados}

En respuesta al primer objetivo se presentan los resultados del trabajo de gabinete: la descripción del concepto de autorregulación y el análisis de la actividad de Autocontrol, el sistema de autorregulación publicitaria español. En respuesta al segundo objetivo se presentan los resultados de las entrevistas realizadas para conocer cómo gestionan las principales agencias de publicidad la supervisión ética y legal de su actividad.

\subsection{Concepto y elementos constitutivos de los siste- mas de autorregulación}

La relevancia económica y social de la publicidad exige su control por parte de los poderes públicos mediante la apro- la publicidad, con la finalidad de imponerse a sí mismos, de forma voluntaria, una norma en materia publicitaria (Patiño-Alves, 2007). Desde un punto de vista más práctico, se define como un sistema voluntariamente establecido por las empresas que integran el sector publicitario (anunciantes, agencias y medios de comunicación) a través del cual se procura que la publicidad se lleve a cabo de forma legal, honesta y responsable, en beneficio de los consumidores, de los competidores, del mercado publicitario y de la sociedad en general (Fernando-Magarzo, 2008).

Para que un sistema de autorregulación publicitaria se configure como tal y sea efectivo debe observar cuatro elementos constitutivos (Gómez-Segade; Lema Devesa, 1981):

- existencia de una agrupación voluntaria de miembros que participen activa y pasivamente en la industria publicitaria;

- que el grupo se imponga unas normas de carácter moral o ético -los denominados códigos de conducta publicitaria-que deberán ser observadas por todo el colectivo que integra el sistema;

- existencia de un órgano de control y supervisión -caracterizado por la especialización publicitaria y la imparcialidad de sus miembros- que resuelva controversias;

- que este órgano de control tenga carácter sancionador y capacidad para ejecutar sus decisiones con respecto a los miembros adheridos voluntariamente al sistema.

La autorregulación constituye un sistema de control de la publicidad más amplio, detallado y efectivo que la regulación, pues surge de la libre voluntad de los agentes implicados quienes, sin renunciar a la libertad creativa y de expresión, asumen un compromiso de responsabilidad en el desempeño de su actividad profesional (Megías-Quirós; Cabrera-Cano, 2013). A diferencia de los sistemas anglosajones -en los que la autorregulación emerge con el fin de anticiparse a la regulación pública-, en Europa la autorregulación surge en un contexto en el que ya existe una legislación estatal, por lo que en su existencia y consolidación también incide la falta de eficacia del ordenamiento jurídico vigente y el impulso de la autorregulación por parte del legislador (Patiño-Alves, 2007).

En Europa la autorregulación publicitaria surge tras la aprobación en 1937 del Código de prácticas publicitarias y mercadotecnia de la International Chamber of Commerce y de 
la Resolución del Consejo de Europa, de 18 de febrero de 1972, sobre protección de los consumidores. Esta Resolución, que inició el reconocimiento e impulso legal de la autorregulación, recomendaba a los estados la creación de organismos de autodisciplina y su cooperación con organismos públicos y asociaciones de consumidores. En 1992 la industria publicitaria europea avanzó en su compromiso ético mediante la creación de la European Advertising Standards Alliance, un ente concebido para promover la autorregulación publicitaria en los países de Europa.

Tras reconocer y fomentar los sistemas de autorregulación, el legislador ha ido reconociendo expresamente la legitimidad de sus organismos extrajudiciales de resolución de controversias de una manera progresiva, hasta el punto de llegar a integrar los sistemas de autorregulación publicitaria y, más concretamente, sus códigos de conducta en la actual legislación -comunitaria y estatal- en materia de competencia desleal (Fernando-Magarzo, 2008; VilajoanaAlejandre, 2011).

Desde un punto de vista práctico, además de evidenciar un compromiso ético, los sistemas de autorregulación se configuran como un efectivo complemento a la regulación publicitaria. Frente a la mayor seguridad que proporcionan las normas jurídicas, la reconocida eficacia y eficiencia de los sistemas de autorregulación (Boddewyn, 1985) deriva de su mayor rapidez, especialización, transparencia, bajo coste y fácil acceso (Patiño-Alves, 2007).

\section{Desde su creación en 1995, Autocontrol ha revisado más de 158.000 anuncios previamente a su difusión}

\subsection{Autocontrol: el sistema de autorregulación publi- citaria español}

Los primeros antecedentes en materia de autorregulación publicitaria en España se remontan a 1965, cuando los profesionales elaboraron los primeros textos que recogían el compromiso ético de su actividad. Tras el fracaso de los primeros proyectos, en 1976 anunciantes, agencias y medios impulsaron con el apoyo de la Administración, la constitución de Autocontrol de la Publicidad S.A., el primer organismo de autorregulación publicitaria español. Con la voluntad de superar la defectuosa organización y la cuestionada independencia e imparcialidad de este organismo, en 1995 se constituyó la Asociación de Autocontrol de la Publicidad, actualmente denominada Autocontrol, Asociación para la Autorregulación de la Comunicación Comercial.

http://www.autocontrol.es

Autocontrol es una asociación sin ánimo de lucro cuyo principal objetivo es gestionar el sistema español de autorregulación publicitaria. Integrada voluntariamente por los principales anunciantes, agencias, medios de comunicación y asociaciones relacionadas con la actividad publicitaria en España, cuenta con 500 socios directos y alrededor de 2.000 indirectos, por lo que se estima que representa a más del 70 por ciento de la inversión publicitaria (Román-Domínguez; Gómez-Castallo, 2016).

La actividad de Autocontrol responde a tres objetivos básicos:

- contribuir a la ética y la lealtad de la publicidad en beneficio de los consumidores;

- verificar el cumplimiento de las normas legales y deontológicas;

- procurar la pacífica y rápida resolución de reclamaciones.

Autocontrol gestiona el sistema de autorregulación publicitaria sobre la base de tres instrumentos:

- sus propios códigos éticos;

- sistema de asesoramiento previo;

- constitución de un órgano independiente, el Jurado de la Publicidad, encargado de verificar la aplicación de los códigos éticos y resolver controversias.

Entre los códigos aprobados por Autocontrol destacan:

- Código de conducta publicitaria, de carácter general y cuya primera versión se aprobó en 1996;

- Código ético de comercio electrónico y publicidad interactiva, en vigor desde 2003; 


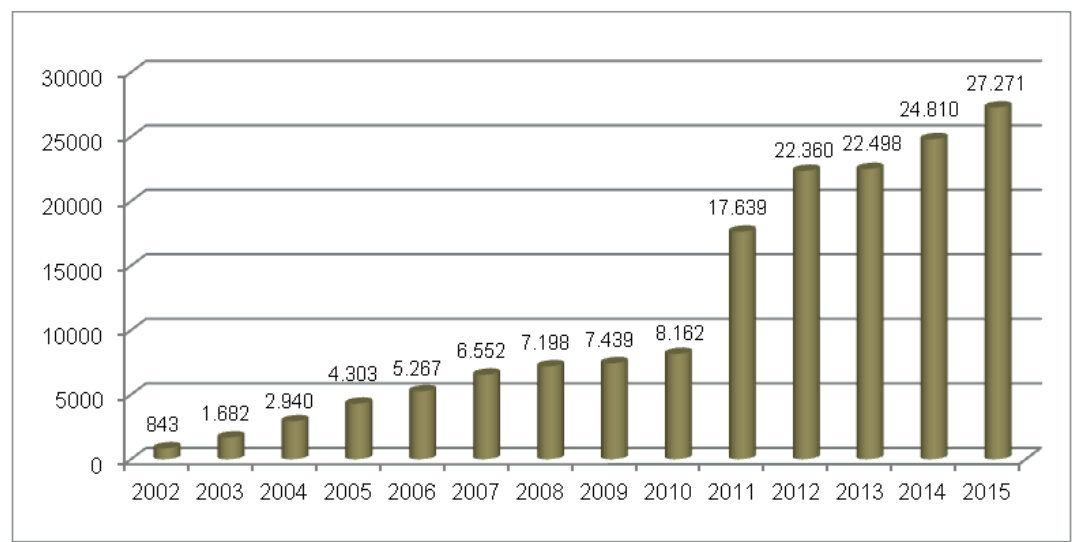

Gráfico 1. Histórico de la actividad preventiva de Autocontrol: consultas y Copy Advice ${ }^{\circledR}$ tración Pública, con el objetivo de dotar de una mayor objetividad y transparencia al sistema y, sobre todo, dotarlo de una capacidad sancionadora más efectiva.

Entre estos convenios destaca por ejemplo el Acuerdo para el fomento de la corregulación sobre las comunicaciones comerciales en televisión firmado por la Comisión Nacional de los Mercados y la Competencia -la actual autoridad audiovisual estatal- y Autocontrol en 2015. Mediante este acuerdo, que renueva el original de 2003, la Administración reconoce el sistema de autorregulación de la publicidad televisiva suscrito por los principales operadores en 2002, valora el

- un significativo número de códigos éticos sectoriales, consultables desde la web de Autocontrol.

Desde 2001 Autocontrol vehicula su servicio de consultas previas principalmente a través del denominado Copy $\mathrm{Ad}$ vice $^{\circledast}$. Este servicio ofrece a anunciantes, agencias y medios un asesoramiento voluntario, confidencial y no vinculante, sobre la corrección legal y ética de un proyecto de campaña o anuncio previo a su difusión.

En relación con el control a posteriori de la publicidad, el Jurado es el órgano independiente encargado de aplicar los códigos éticos y de resolver extrajudicialmente controversias. Compuesto por expertos independientes y de reconocido prestigio, una cuarta parte de sus miembros son nombrados a propuesta de la Agencia Española de Consumo, Seguridad Alimentaria y Nutrición (el organismo que, desde 2014 integra y desempeña las funciones del anterior Instituto Nacional de Consumo). Cualquier persona con un interés legítimo puede presentar una reclamación ante $A u$ tocontrol por infracción de sus códigos éticos. Las resoluciones del Jurado, siempre motivadas, en el caso de confirmar la infracción pueden:

- declarar la incorrección de la publicidad;

- instar al anunciante a la rectificación o el cese de la misma;

- contemplar una amonestación e incluso la difusión específica de la resolución, a pesar de que todas las resoluciones del Jurado se publican en la revista de Autocontrol y en su web.

Dado el carácter voluntario de Autocontrol, la asociación cumple todos los requisitos constitutivos de un sistema de autorregulación. Además Autocontrol ha llevado a cabo una activa política de colaboración con varios organismos de la Administración concretada en la firma de más de 21 convenios, llegando en algunos casos a convertirse en un sistema de corregulación, un mecanismo de control híbrido entre la autorregulación propia del sector y la regulación legal. En los sistemas de corregulación publicitaria la propia industria acoge en su seno a un tercero ajeno a la actividad publicitaria, la Adminis- sistema de Copy Advise ${ }^{\circledR}$ y crea una Comisión mixta para el seguimiento de su aplicación.

\subsubsection{Balance de actividad}

Autocontrol publica anualmente una memoria de actividad. Desde su creación en 1995 ha atendido más de 158.000 consultas previas y en paralelo ha resuelto más de 3.270 reclamaciones a través del Jurado de la Publicidad (RománDomínguez; Gómez-Castallo, 2016).

El gráfico 1 muestra la evolución de la actividad preventiva de Autocontrol desde 2002, año en el que al tradicional servicio de consultas jurídicas o deontológicas se unió el Copy Advice $^{\circledR}$. De las 27.271 solicitudes de asesoramiento previo recibidas en 2015, 21.716 se tramitaron a través del Copy Advise $^{\circledR}$. De éstas, un $85 \%$ obtuvo una respuesta positiva, en el $11,4 \%$ de los casos se recomendaron modificaciones y tan sólo en un 3,5\% de los casos ser desaconsejó la difusión de la publicidad.

Entre los beneficios del progresivo incremento de consultas previas, Autocontrol destaca la reducción del riesgo de incumplimiento de las normas deontológicas y legales, y su utilidad a la hora de aplicar normas de difícil interpretación.

El gráfico 2 muestra la evolución en el número de reclamaciones sobre publicidad difundida tramitadas por Autocontrol en los últimos 10 años. De las 305 reclamaciones recibidas en 2015, 93 fueron resueltas por mediación o acuerdo, sin requerir la intervención del Jurado; de los 212 casos que sí requirieron su intervención, 74 fueron estimados -total

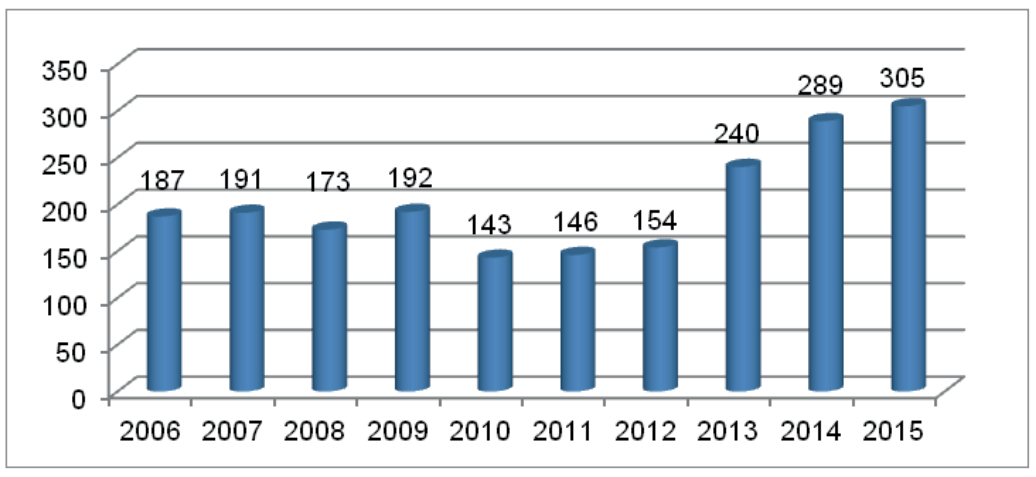

Gráfico 2. Histórico del control a posteriori de Autocontrol: reclamaciones 2006-2015 
o parcialmente- y 84 fueron desestimados. Tras un período de estabilización motivada en gran medida por el incremento de su labor preventiva, en los últimos años se constata un incremento del número de reclamaciones. Esto se puede deber, entre otras cuestiones, al mayor conocimiento de $\mathrm{Au}$ tocontrol por parte de los consumidores fruto de sus propias campañas de publicidad.

Tabla 2. Codificación por categorías

\begin{tabular}{|c|c|c|c|}
\hline $\mathbf{N}$ & Categoría & Items & Frec. \\
\hline C.1. & Incidencia ética y legislación & $\begin{array}{l}\text { Alta } \\
\text { Moderada }\end{array}$ & $\begin{array}{l}6 \\
2\end{array}$ \\
\hline C.2. & Vía de supervisión & $\begin{array}{l}\text { Interna en agencia } \\
\text { Autocontrol } \\
\text { Externa habitual bufete/s especializado/s } \\
\text { Externa puntual bufete especializado } \\
\text { Departamento jurídico anunciante }\end{array}$ & $\begin{array}{l}3 \\
8 \\
7 \\
1 \\
8\end{array}$ \\
\hline C.3. & Gestión interna de la supervisión ética y legal & $\begin{array}{l}\text { Fase previa a ejecución } \\
\text { Surge en el proceso } \\
\text { Perfiles profesionales: cuentas }\end{array}$ & $\begin{array}{l}5 \\
2 \\
6\end{array}$ \\
\hline C.4. & Formación continua & $\begin{array}{l}\text { Interna, puntual } \\
\text { Externa, asistencia puntual a seminarios } \\
\text { Aspecto a mejorar }\end{array}$ & $\begin{array}{l}3 \\
7 \\
3\end{array}$ \\
\hline C.5. & Autorregulación & $\begin{array}{l}\text { Adhesión Autocontrol } \\
\text { Uso Copy Advice } \\
\text { Valoración positiva }\end{array}$ & $\begin{array}{l}6 \\
8 \\
7\end{array}$ \\
\hline
\end{tabular}

En el contexto europeo Autocontrol fue en 2015 el segundo organismo de Europa con mayor número de Copy Advice ${ }^{\circledR}$ emitidos, por detrás del consolidado y eficaz sistema de autorregulación del Reino Unido y por delante de Francia, Alemania y Hungría. En relación con el número de reclamaciones tramitadas, Autocontrol ocupa la décima posición en el ranking europeo de reclamaciones, muy por detrás del sistema británico, que lidera la clasificación con un total de 27.183 reclamaciones gestionadas (EASA, 2016).

Pese al evidente compromiso ético y la relevante actividad consolidada por Autocontrol, desde un punto de vista crítico algunos estudios han identificado carencias en el

\section{Algunos estudios identifican carencias en la independencia y efectividad de Autocontrol}

sistema, principalmente en relación con los criterios de independencia y efectividad. Patiño-Alves (2007) alerta del amplio y excesivo poder del que dispone la Junta Directiva de Autocontrol -la cual no deja de representar los intereses económicos de los colectivos adheridos- ya que, entre otras funciones, escoge el 75 por ciento de los miembros del Jurado, aprueba su Reglamento y ejecuta las medidas y sanciones derivadas de sus resoluciones. Para Martín-Llaguno y Hernández-Ruiz (2009) dos son las principales limitaciones del sistema:

- contemplar únicamente la publicidad con finalidad comercial;

- su actuación sólo a instancia de parte, reservando el control de oficio a los acuerdos puntuales con las administraciones públicas.

Tras realizar un análisis comparativo entre la configuración y actividad de Autocontrol y de la Advertising Standards Authority -el sistema de autorregulación británico cuyo modelo es referencia internacional-, Muela-Molina y PerellóOliver (2014) concluyen que las principales debilidades de

Autocontrol son su efectividad e independencia. La ausencia de un servicio de monitorización de la publicidad contraria a los principios éticos sobre todo, de un servicio de supervisión del cumplimiento de las resoluciones del Jurado, cuestionan según estos autores su efectividad. Asimismo, la excesiva influencia de la Junta Directiva de Autocontrol en la configuración del sistema cuestiona su independencia y recomienda una mayor presencia e involucración de la Administración y los consumidores.

\subsection{Gestión e incidencia práctica del condicionante ético y legal en las agencias de publicidad}

El análisis del contenido de las entrevistas a directivos de agencias de publicidad permitió identificar las cinco categorías de análisis recogidas en la tabla 2. Esta tabla también incorpora las cuestiones relevantes que se advirtieron al analizar los datos de cada categoría y la frecuencia con la que los items identificados aparecen en la transcripción de las entrevistas.

En el proceso de categorización, además de las categorías esperadas -inducidas por el propio guion de la entrevista-, surgieron otras inesperadas en relación con los objetivos planteados. Al codificar se observó que los datos obtenidos saturaron en la mayoría de casos las diferentes categorías, lo que constató que la muestra final cumple con los objetivos de la investigación. Para presentar los resultados seguiremos la estructura planteada en la tabla 2.

\subsubsection{Incidencia de condicionantes ético y legal}

En relación con la incidencia de los códigos éticos y la legislación en la publicidad, los profesionales los perciben como un condicionante más de su actividad. Los entrevistados manifiestan mayoritariamente que el marco ético y legal condiciona considerablemente la creatividad de las campañas, conscientes de que un descuido puede impedir la difusión o propiciar la retirada posterior de la publicidad, con el consiguiente coste económico y en términos de reputación.

Los publicitarios son conscientes de los restrictivos límites que en algunos sectores específicos imponen los propios 
anunciantes a través de los códigos éticos de Autocontrol. Más allá de la regulación y la autorregulación, los publicitarios son conscientes del control social que ejercen los propios consumidores y cómo estos pueden amplificar su malestar ante la publicidad contraria a principios éticos por diversos medios, como por ejemplo las redes sociales, con los riesgos que ello implica.

\subsubsection{Vías de supervisión ética y legal de la publicidad}

En cuanto a la supervisión interna de la adecuación ética y legal de las campañas, se identifican cuatro vías no excluyentes de supervisión:

- interna, llevada a cabo en la propia agencia;

- consulta a Autocontrol mediante solicitud de Copy Advice ${ }^{\circledR}$;

- consulta externa a abogados especializados;

- supervisión externa por parte del departamento jurídico del anunciante.

En relación con la supervisión interna, cabe destacar la escasa presencia de un departamento especializado en ética y derecho publicitario en las propias agencias, así como la mayor presencia de una figura -adscrita al departamento de administración o finanzas- encargada de gestionar y supervisar temas de contratación y, en algunos casos, también coordinar la supervisión ética y legal.

Las agencias de publicidad confían mayoritariamente en Autocontrol para la supervisión ética y legal de su actividad

Las agencias confían mayoritariamente en Autocontrol para la supervisión ética y legal de sus campañas, especialmente en aquellos sectores sujetos a códigos éticos específicos que, como en el caso de algunas bebidas alcohólicas, exigen a los anunciantes solicitar siempre el Copy Advice ${ }^{\circledR}$. La mayoría de las agencias contemplan la posibilidad de que esta solicitud la gestione, bien la propia agencia o bien el propio anunciante, según el caso.

Todas las agencias consultadas disponen de abogados externos -mayoritariamente especializados en derecho de la publicidad- con los que colaboran, de manera habitual o puntual, para resolver dudas específicas. Entre las agencias que disponen de una colaboración externa estable, una de ellas dispone de un abogado externo pero dedicado prácticamente en exclusiva a asesorarles y a pesar de ello, en paralelo la agencia consulta por otras vías, principalmente Autocontrol.

La mayoría de las agencias que recurren habitualmente a un despacho de abogados también contemplan la posibilidad de consultar puntualmente con despachos todavía más especializados dudas concretas, como los acuerdos con celebrities, temas musicales, promociones, etc. Las agencias que con carácter general formulan consultas puntuales a sus despachos de referencia recurren a ellos cuando detectan temas controvertidos por su novedad, su especialización o por tratarse de anunciantes sujetos a regulación y a códigos de conducta específicos, en la mayoría de casos más restrictivos que la propia legislación.
Por último, todas las agencias contemplan como vía de supervisión adicional el departamento legal de sus clientes, presente habitualmente en multinacionales y grandes anunciantes. La colaboración entre el departamento jurídico interno del anunciante y los abogados de la agencia es frecuente cuando surgen dudas, aunque la mayoría de los entrevistados destacan la poca especialización de estos departamentos y el riesgo de que las consultas ralenticen el proceso publicitario.

\section{Las agencias supervisan la adecuación ética y legal de la publicidad por una tri- ple o cuádruple vía}

\subsubsection{Gestión interna de la supervisión ética y legal}

La mayoría de las agencias incorpora la revisión ética y legal en el proceso creativo previo a la producción de la publicidad. Si el producto está sujeto a restricciones específicas, éstas se apuntan ya en el briefing $y$, en cualquier caso, las agencias intentan que no se presente nada al cliente sin una revisión previa, aunque a posteriori, cuando el anuncio esté terminado, alguna de ellas solicite el Copy Advice ${ }^{\circledR}$ definitivo. El departamento de cuentas de la agencia es el que asume la responsabilidad de detectar e identificar problemas mediante la supervisión del esbozo o guion elaborado por el equipo creativo, que acostumbra a tener presentes sólo las cuestiones éticas y legales de carácter básico y general.

\subsubsection{Formación continua}

La formación continua en materia de ética y legislación publicitaria es un tema pendiente en las agencias, incompatible en muchos casos, con las dinámicas y rutinas de su día a día. Esta carencia se suple con la consulta de material y asistencia puntual a actos organizados por Autocontrol, con el apoyo de los abogados externos y con la propia experiencia adquirida en la agencia.

\subsubsection{Valoración, adhesión y trascendencia práctica de Au- tocontrol}

Todas las agencias manifiestan conocer Autocontrol, valorar positivamente su actividad y sobre todo utilizar el servicio de Copy Advice ${ }^{\circledR}$.

La mayoría son miembros de Autocontrol y las que no están adheridas al sistema, contratan puntualmente el sistema de Copy Advice ${ }^{\circledR}$.

En todos los casos el Copy Advice ${ }^{\circledR}$ se utiliza como una vía complementaria para garantizar la adecuación legal y ética de las campañas, aunque no todas las piezas lo pasan. Acostumbra a solicitarse en paralelo a la consulta con el abogado o despacho de referencia. Se solicita en tres supuestos:

- cuando surgen dudas;

- cuando el sector al que pertenece el anunciante está sujeto a códigos éticos específicos;

- cuando es el propio anunciante el que pide a la agencia pasar el Copy Advice ${ }^{\circledR}$ en todas sus campañas.

Todas las agencias consideran correcto el servicio de Copy Advice ${ }^{\circledR}$, en líneas generales acorde a lo que se espera de un 
servicio de sus características. Algunas respuestas señalan que los informes de Copy Advice ${ }^{\circledR}$ acostumbran a identificar los problemas y resolver dudas, pero carecen de una propuesta de solución, un aspecto que sería muy valorado por los profesionales de la publicidad.

\section{Discusión y conclusiones}

Desde un punto de vista general podemos concluir que los profesionales de la publicidad muestran un elevado compromiso ético en el desempeño de su actividad. La creación de un sistema de autorregulación propio -impulsado voluntariamente por los agentes implicados en la publicidad-, su configuración -en línea con los requisitos básicos para garantizar su efectividad-y el balance de los más de 20 años de actividad de Autocontrol, así lo constatan.

En cumplimiento de sus funciones, Autocontrol ha desempeñado una relevante labor de asesoramiento previo y control a posteriori de la publicidad, que lo avala como un actor clave para el fomento y garantía del desarrollo de una publicidad ética en España. A raíz del éxito de su servicio de Copy Advice ${ }^{\circledR}$ y de los acuerdos que Autocontrol ha suscrito en los últimos años con la Administración en sectores y medios específicos -por la vía del compromiso ético o por la de la corregulación-, los datos publicados por Autocontrol evidencian que una gran parte de la publicidad que se difunde en España pasa en mayor o menor medida por una exhaustiva supervisión ética y legal.

Una gran parte de la publicidad que se difunde en España pasa en mayor o menor medida por una exhaustiva supervisión ética y legal

Conscientes del impacto social de su actividad, los profesionales de las principales agencias de publicidad que operan en España dotan de un gran valor y relevancia el desarrollo ético de su actividad. Esta conclusión se constata en tanto que las agencias supervisan la adecuación ética y legal de sus campañas por una triple o cuádruple vía que comprende a Autocontrol como vía principal y necesaria, aunque no suficiente en materia de supervisión legal, que no estrictamente ética, de la publicidad.

A pesar de los estudios que evidencian la necesidad y pertinencia de incrementar la independencia y eficacia del sistema, el nivel de compromiso ético del sector se evidencia al constatar que en los últimos cinco años Autocontrol ha revisado, con carácter previo a su difusión, más de 98.000 anuncios publicitarios, de los cuales tan sólo en un $9 \%$ de los casos recomendó modificaciones y en un 3\% desaconsejó su difusión. Si entendemos que el verdadero propósito de la autorregulación publicitaria es más moral y ético que disciplinario (Boddewyn, 1989), podemos concluir que la industria publicitaria española vela por garantizar, de una manera práctica, que su actividad se ajusta a los principios éticos recogidos en los propios códigos de Autocontrol.

La relevancia de la intersección entre ética y publicidad, y la trascendencia práctica de los sistemas de autorregulación jus- tifican la necesidad de nuevos estudios que profundicen en el compromiso ético de la publicidad desde una perspectiva transversal. Contemplar al resto de los agentes implicados en la actividad publicitaria, analizar el contenido de los códigos éticos para valorar sus compromisos más allá de los límites que impone la legislación y plantear nuevos trabajos que analicen y comparen modelos para optimizar los existentes, son algunos de los futuros retos que plantea esta investigación.

\section{Bibliografía}

Aznar, Hugo (2000). "Publicidad y ética: la vía de la autorregulación". Revista latina de comunicación social, n. 25. http://www.ull.es/publicaciones/latina/aa2000yen/148hugoaznar. html

Boddewyn, Jean J. (1985). "Advertising self-regulation: Private government and agent of public policy". Journal of public policy \& marketing, v. 4, n. 1, pp. 129-141.

Boddewyn, Jean J. (1989). “Advertising self-regulation: True purpose and limits". Journal of advertising, v. 18, n. 2, pp. 19-27.

https://goo.gl/QF89ww

Daymon, Christine; Holloway, Immy (2011). Qualitative research methods in public relations and marketing communications. New York: Routledge. ISBN: 9780415471183 https://goo.gl/JuMTcs

Drumwright, Minette E.; Murphy, Patrick E. (2004). “How advertising practitioners view ethics". Journal of advertising, v. 33, n. 2, pp. 7-24.

http://class.classmatandread.net/regulation/-ethics_followUp.pdf

Drumwright, Minette E.; Murphy, Patrick E. (2009). "The current state of advertising ethics". Journal of advertising, $v$. 38, n. 1, pp. 83-107.

https://goo.gl/tgZMUS

https://doi.org/10.2753/JOA0091-3367380106

European Advertising Standards Alliance (EASA) (2016). European trends in advertising complaints, copy advice and pre-clearance 2015.

http://www.easa-alliance.org/products-services/publications/ statistics

Eguizábal, Raúl (2010). Teoría de la publicidad (2a ed.). Madrid: Cátedra. ISBN: 8437623944

Fernando-Magarzo, María-Rosario (2008). "La consolidación de la autorregulación en España: fomento normativo y reconocimiento jurisprudencial". Autocontrol, n. 135, pp. 18-30.

Gómez-Castallo, José-Domingo; Fernando-Magarzo, María-Rosario (2010). "Códigos de conducta y sistemas de autorregulación: en especial, en el ámbito de las comunicaciones comerciales". Comunicaciones en propiedad industrial y derecho de la competencia, n. 58, pp. 135-158.

Gómez-Segade, José-Antonio; Lema-Devesa, Carlos (1981). "La autodisciplina publicitaria en el derecho comparado y en el derecho español". Actas de derecho industrial, n. 7, pp. 31-114. 
Hyman, Michael R.; Tansey, Richard; Clarck, James W. (1994). "Research on advertising ethics: Past, present and future". Journal of advertising, v. 23, n. 3, pp. 5-15.

https://goo.gl/IAofW6

http://dx.doi.org/10.1080/00913367.1994.10673446

Ipmark (2014). "McCann lidera el ranking de agencias de publicidad". Ipmark, 14 octubre.

http://ipmark.com/mccann-lidera-ranking-agencias

Ipmark (2016). "McCann lidera el ranking de agencias de publicidad por inversión gestionada". Ipmark, 15 diciembre. http://ipmark.com/mccann-lidera-ranking-agenciasagencias-inversion-gestionada

Krugman, Dean; Ferrell, O. C. (1981). "The organizational ethics of advertising: corporate and agency views". Journal of advertising, v. 10, n. 1, pp. 21-48.

https://goo.gl/RM8hql

http://dx.doi.org/10.1080/00913367.1981.10672752

Lema-Devesa, Carlos; Gómez-Montero, Jesús (2010). Código de publicidad (5a ed.). Madrid: Marcial Pons. ISBN: 978 8497687973

Lipovetsky, Gilles (1994). El crepúsculo del deber. La ética indolora de los nuevos tiempos democráticos. Barcelona: Anagrama. ISBN: 9788433913784

Martín-Llaguno, Marta; Hernández-Ruiz, Alejandra (2009). “El control de la comunicación comercial en un mundo globalizado: regulación, autorregulación e hiperregulación de la publicidad". Portal de la comunicación InCOM-UAB. Lecciones.

http://www.portalcomunicacion.com/lecciones_det. asp?id $=50$

Martín-Llaguno, Marta; Hernández-Ruiz, Alejandra (2010). "Efectos de la deontología de la publicidad en la actitud profesional". Questiones publicitarias, v. 1, n. 15, pp. 99-113. http://rua.ua.es/dspace/handle/10045/25701
Massaguer-Fuentes, José (2011). “Códigos de conducta y competencia desleal: aspectos sustantivos y procesales". Indret: Revista para el análisis del derecho, n. 2, pp. 1-39. http://www.indret.com/pdf/823_es.pdf

Megías-Quirós, José-Justo; Cabrera-Caro, Leticia (2013). Ética y derecho en la publicidad. Granada: Comares. ISBN: 9788490451182

Muela-Molina, Clara; Perelló-Oliver, Salvador (2014). “Advertising self-regulation. A comparative analysis between the United Kingdom and Spain". Comunication \& society, v. 27, n. 3, pp. 1-18.

http://www.unav.es/fcom/communication-society/es/ resumen.php?art_id $=498$

Patiño-Alves, Beatriz (2007). La autorregulación publicitaria: especial referencia al sistema español. Barcelona: Bosch. ISBN: 9788497902939

Román-Domínguez, Almudena; Gómez-Castallo, JoséDomingo (2016). Autocontrol de la publicidad. Balance de actividad 2015.

http://www.autocontrol.es/pdfs/balance_15_AUTOCONTROL. $p d f$

Sánchez-Guzmán, José-Ramón (1993). Teoría de la publicidad (2a ed.). Madrid: Tecnos. ISBN: 8430923829

Taylor, Ronald E. (2002). "Inside the Asociación de Autocontrol de la Publicidad: A qualitative study of advertising selfregulation in Spain". Journal of intercultural communication research, v. 31, n. 3, pp. 181-190.

Vilajoana-Alejandre, Sandra (2011). Las leyes de la publicidad: límites jurídicos de la actividad publicitaria. Barcelona: UOC. ISBN: 9788497884259

Vilches, Lorenzo (coord.) (2011). La investigación en comunicación: métodos y técnicas en la era digital. Barcelona: Gedisa. ISBN: 9788497846691

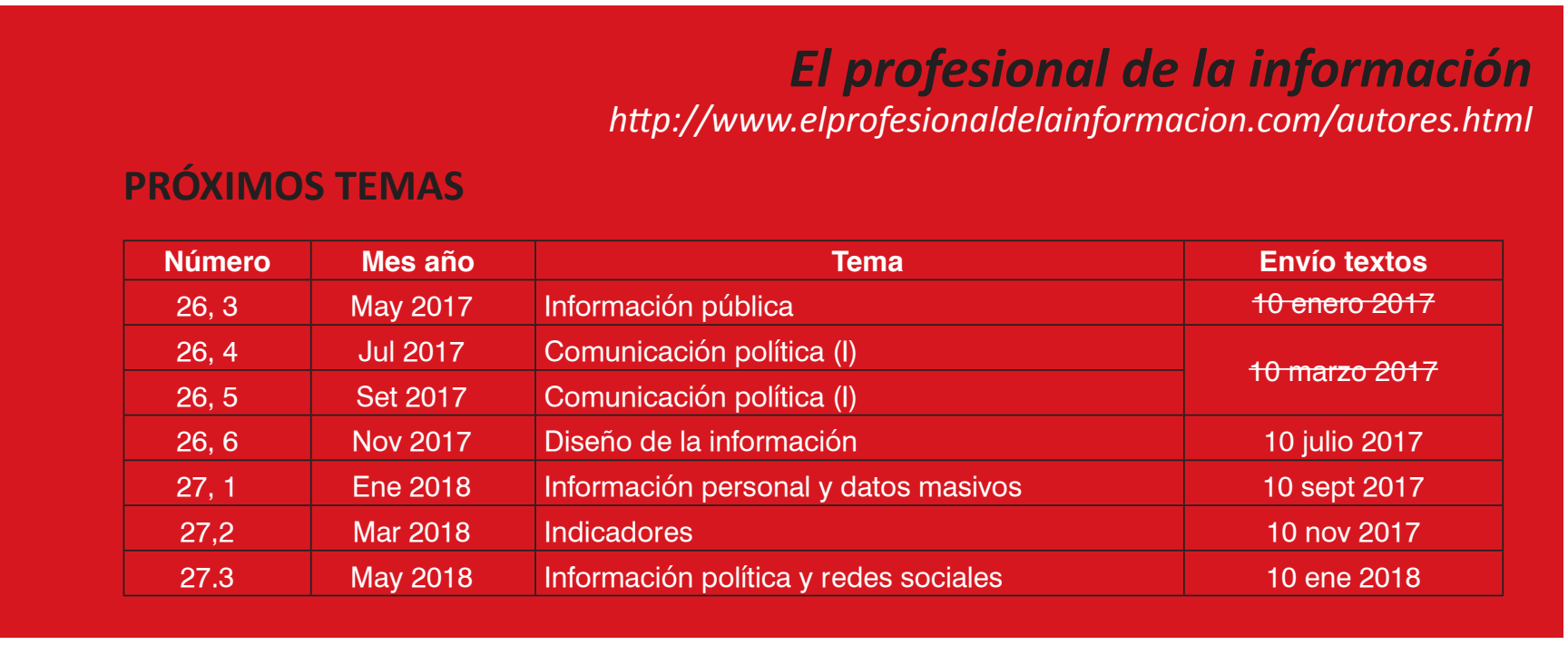

